\title{
Restricciones del entorno a la competitividad de PyMEs industriales en México
}

\author{
Restrictions of the environment on the competitiveness of \\ Industrial SMEs in Mexico
Lizeth V. Jiménez-Martínez ${ }^{a}$, Yacsiri Y. Cerón-González ${ }^{b}$, Jaqueline J. Zavala-Arce ${ }^{c}$, Zaira L. González-Gómez ${ }^{d}$

\begin{abstract}
:
Industrial Small and Medium-sized Enterprises (SMEs) in Mexico have an outstanding performance in the national economy, since the creation of new policies focused on their development aims at economic and competitive growth. Such is the case, that according to INEGI results, more than 4.1 million SMEs are registered in Mexico, however, there are various gaps that hinder the development of the organizations belonging to this classification.

Many determining factors are considered for the introduction of an SME in the competitive environment, which at the same time present a barrier to its development. Examples of this are the use of competitive context analysis tools, financing or raising capital, use and management of technologies and information, implementation of public policies, human capital management, innovations and increased productivity, which represent complications with an impact in the success of organizations. For this reason, this article presents a critical and constructive analysis, based on the synthesis, classification, comparison and interpretation of previous publications, with the aim of identifying the various aspects of restriction to that industrial SMEs face in the competitive environment in Mexico.
\end{abstract}

Keywords:

Industrial SMEs, Competitive restrictions, Competitive Environment, Competitiveness

\section{Resumen:}

Las Pequeñas y Medianas Empresas (PyMEs) industriales en México tienen un destacado desempeño en la economía nacional, ya que la creación de nuevas políticas enfocadas en su desarrollo tiene como objetivo el crecimiento económico y competitivo. Tal es el caso, que, según resultados de INEGI, en México se encuentran registradas más de 4.1 millones de PyMEs, sin embargo, existen diversas brechas que obstaculizan el desarrollo de las organizaciones pertenecientes a esta clasificación.

Se consideran diversos factores determinantes para la introducción de una PyME en el entorno competitivo, que a la vez presentan una barrera para su desarrollo. Ejemplos de esto son la utilización de herramientas de análisis del contexto competitivo, financiamiento u obtención de capital, uso y gestión de las tecnologías de información, implementación de políticas públicas, gestión del capital humano, innovaciones e incremento de productividad, que representan obstáculos con impacto en el éxito de las organizaciones. Por tal motivo, en este artículo se presenta una recopilación documental, a partir de la síntesis, clasificación, comparación e interpretación de publicaciones previas, que tiene como objetivo observar las diversas vertientes de las restricciones a las que se enfrentan las PyMEs industriales en el entorno competitivo en México.

\section{Palabras Clave:}

PyMEs industriales, Restricciones de competitivas, Entorno Competitivo, Competitividad

\footnotetext{
a Universidad Autónoma del Estado de Hidalgo, https://orcid.org/0000-0003-0410-0079, email: ji362591@uaeh.edu.mx

b Universidad Autónoma del Estado de Hidalgo, https://orcid.org/0000-0002-6994-6887, email: ce362590@uaeh.edu.mx

c Universidad Autónoma del Estado de Hidalgo, https://orcid.org/0000-0003-2654-0753, email: za361284@uaeh.edu.mx

d Autor de Correspondencia, Universidad Autónoma del Estado de Hidalgo, https://orcid.org/0000-0003-1688-2581, email: go354615@uaeh.edu.mx
} 


\section{Introducción}

Las pequeñas y medianas empresas (PyMEs) se han convertido en un tema sobresaliente dentro del ámbito socioeconómico, por lo que entender su comportamiento en un entorno competitivo resulta un tópico relevante de investigación. En la actualidad, el entorno en el que se desarrollan las PyMEs se encuentra enfocado al crecimiento competitivo de las organizaciones como medio de progreso económico, ya que el entomo empresarial proporciona a la economía un bienestar productivo, que es la forma de sustento de las actividades de la población (Gonzalez \& Herrera, 2011), siendo uno de los principales proveedores de las economías modernas (Pazos \& Lopez, 2007); sin embargo, la introducción o permanencia de este conjunto de empresas resulta difícil por la serie de problemas que presentan en su consolidación o desarrollo en el entorno competitivo. Por lo anterior, es fundamental conocer los obstáculos más importantes al desarrollo competitivo de las PyMEs, identificando los factores tanto internos como externos, que están obligadas a analizar, dado que influyen en su competitividad (Martinez \& Mejia, 2013).

En cuanto a la concepción de las PyMEs, resulta difícil establecer un concepto, dada la gran variedad que de ellos existe; sin embargo, pueden considerarse aspectos de heterogeneidad mundial (Suarez, Tricas, \& Toledano, 2012) en cuanto a su función principal, que consiste en proveer bienes y servicios básicos a las grandes empresas (Rivas, 2002). La clasificación empleada en México está establecida por la Secretaría de Economía (SE) desde el año de 1985, quien determinó el tamaño de las empresas en función al número de trabajadores, así como el sector al que pertenece. No obstante, una característica importante que refieren las diversas fuentes para determinar el tamaño, es el volumen de ventas (Cardozo, Velasquez, \& Rodriguez, 2012).

La clasificación actual considera ambos criterios, como se muestra en la tabla 1, publicada en el Diario Oficial de la Federación (DOF).

En función de lo anterior, y de acuerdo a sus características, en México la constitución de PyMEs ha experimentado un crecimiento exponencial lo que ha permitido la apertura hacia otros mercados, generando oportunidades y nuevos escenarios para su competitividad (Rodriguez, 2015), considerando que, junto con las microempresas, representan la economía de mayor relevancia y su dinamismo potencializa el crecimiento de su entorno competitivo (Ruiz, 2013). Portal motivo, considerar la competitividad como fase determinante en su desarrollo, otorga a las PyMEs la posibilidad de sobrevivir en el entorno empresarial (Saavedra \& Tapia, 2011)

Tabla 1. Clasificación de las empresas mexicanas publicado en el Diario Oficial de la Federación

\begin{tabular}{|c|c|c|c|c|}
\hline Tamaho & Sector & $\begin{array}{l}\text { Rango de nûmero } \\
\text { de tratsajsders: }\end{array}$ & $\begin{array}{l}\text { Rango de monto do } \\
\text { ventas anuales } \\
\text { (midp) }\end{array}$ & $\begin{array}{l}\text { Tope mixime } \\
\text { combinado" }\end{array}$ \\
\hline Micro & Tẹdas & Hasta 10 & Hasta \$4 & 46 \\
\hline \multirow[t]{2}{*}{ Pequería } & Comercis & Desde 11 hasta 30 & Cesde 34.01 hasta $\$ 100$ & 98 \\
\hline & Indistria y Servicios & Desde 11 hasta 50 & Desde $\$ 4.01$ hasta $\$ 100$ & 56 \\
\hline \multirow[t]{3}{*}{ Medina } & Cometio & Desde 31 hassa 100 & Desde $\$ 100.01$ hasta $\$ 250$ & 235 \\
\hline & Servios & Desde 51 hasta 100 & & \\
\hline & indusfria & Desde 51 hasta 250 & Desde $\$ 10001$ hasta $\$ 250$ & 250 \\
\hline
\end{tabular}

Fuente: (Diario Oficial de la Federación, 2016)

Así entonces, debe tenerse en cuenta que la competitividad consiste en planificar estratégicamente los procesos y productos con base a las necesidades de los clientes o del mercado y las fortalezas o ventajas de la empresa respecto a los competidores, (Canales \& Arnaldo, 2013), además de tener la capacidad de mantener o incrementar su participación en el mercado con un sostenido crecimiento de la productividad (Saavedra \& Milla, 2012), con características que diferencien a la empresa de su competencia, lo que permitirá que se mantengan en el mercado e incrementen su eficacia y eficiencia obteniendo mejores resultados 0 condiciones económicas. (Rodriguez, 2015)

Al respecto, considérese que en México las PyMEs y en general el sector industrial, padecen los efectos de la globalización de la economía y la competitividad (Arroyo, Espinosa, \& Erazo, 2011), debido a que estas empresas se encuentran expuestas a diversas restricciones que obstaculizan su inmersión al entorno empresarial, es decir, la vida de las PyMEs industriales está sujeta a superar los retos de su entorno competitivo (Romero , 2006).

Por lo que se ha expuesto en los párrafos anteriores es que se realiza una revisión documental, con la finalidad de destacar las principales restricciones a que se enfrentan las PyMEs industriales para lograr la competitividad en el entorno empresarial. Para lograr tal propósito, el artículo se estructura partiendo de la conceptualización de la competitividad en las PyMEs como un referente en su desarrollo; se abordan también aspectos sobre su contexto e importancia, y finalmente se plantean los principales obstáculos a los que se enfrenta este tipo de empresas para su introducción o desarrollo en un entomo competitivo. 


\section{PyMEs y Competitividad}

En la actualidad, y debido al rol que desempeñan en el desarrollo económico del país (Diaz, 2006), las PyMEs han empezado a incorporar la competitividad en sus actividades. Si bien es cierto que este término no posee una definición específica, debido a la amplitud de su conceptualización y factores de medición a que está sujeta, a través de los despliegues y enfoques de diversas fuentes, se ha logrado plasmaruna definición que engloba los diferentes factores que la caracterizan.

Al respecto, se puede hacer referencia a lo que sostiene Porter (1991) acerca de que la competitividad de un país se debe a que sus empresas son altamente productivas gracias al uso eficiente de sus recursos humanos, naturales y de capital. De igual manera se alude a lo señalado por la Organización para la Cooperación y el Desarrollo Económico (OCDE), que identifica diversos enfoques sobre competitividad, toman do en consideración la innovación como uno de los principales elementos para el desarrollo económico y las redes de colaboración para fomentar innovaciones. (OCDE, 1992).

Por otra parte, Cabrera, López y Ramírez (2011) la refieren como la factibilidad de una empresa para alcanzar y mantener altos niveles, concentrados en las competencias distintivas o ventajas competitivas que desarrolla internamente, considerando para ello la capacidad para vender más bienes o servicios, y así aumentar su participación en el mercado. Como ejemplo de esto se hace referencia a la elección que se le permite hacer a un grupo de personas entre un vaso de refresco de una marca reconocida mundialmente y una bebida de fabricación casera, donde la mayoría de las personas elegiría la bebida de la marca famosa en función de su posicionamiento o capacidad competitiva en el mercado.

Es necesario en este punto, mencionar que, al referirse a capacidad competitiva, debe considerarse que ésta se desarrolla en el entorno empresarial a través de diversos factores que permiten crear las competencias para generar un entorno de competitividad. Dichos factores son:i) la infraestructura, ii) los mercados financieros, iii) la estructura productiva, iii) la tecnología e innovaciones, por mencionar algunos; (Dussel, 2004) sin embargo, dado que en la actualidad las PyMEs se encuentran interactuando en un entorno globalizado que implica cambios productivos, tecnológicos y financieros, se hace necesario que también el entorno empresarial sea considerado como un factor que contribuye a la competitividad.

Con el propósito de esclarecer este punto, a continuación, se refieren aspectos que permiten la contextualización de las PyMEs industriales mexicanas en el entomo empresarial.

\section{PyMEs Mexicanas}

Las PyMEs han sido objeto de diferentes estudios en México y América Latina; uno de los más relevantes es el realizado por el Instituto Nacional de Geografía y Estadística (INEGl) en el año 2018, donde se muestra que en ese periodo se efectuaron 111,958 registros de este tipo de empresas, las cuales tuvieron una participación del $2.7 \%$ en la economía del país (International Finance Corporation, 2018), generaron el $72 \%$ de empleos formales y contribuyeron en $52 \%$ al Producto Interno Bruto (PIB) (INEGI, 2018), demostrando tener un papel relevante dentro de la actividad económica.

Los datos mencionados ya han sido expresados en estudios realizados con anterioridad (Heradia, 2014) y son una clara evidencia de que las PyMEs en México representan uno de los principales medios para impulsar el desarrollo económico, en tanto que permiten que los recursos estén mejor distribuidos en el territorio nacional.

A diferencia de las microempresas, consideradas en su mayoría como unidades económicas de subsistencia, la Comisión Económica para América Latina y el Caribe (CEPAL) señala que "las PyMEs funcionan y operan con criterios de acumulación y buscan un desarrollo empresarial" (CEPAL , 2018), por lo que obligadamente requieren considerar el incremento de su capacidad competitiva, y específicamente dentro del sector industrial explotar las propiedades que tienen para su crecimiento y desarrollo (Saavedra \& Milla, 2012) entre las que se encuentran:

- La experiencia y formación del empresario,

- Capital intelectual valioso,

- La inversión en innovación,

- La importancia de las ideas del fundador,

- La existencia de estructuras formales e informales de organización,

- La estrategia de la empresa,

- Aspiraciones, audacia y beneficios del propietario,

- Tecnología media y alta,

- El crecimiento como un proceso.

Lo anterior debido a la generación de empleos, la producción de bienes y servicios, el fomento a la investigación y el desarrollo tecnológico, características propias de este tipo de empresas. Sin embargo, es importante también mencionarque únicamente el $25 \%$ de PyMEs mexicanas sobreviven a los dos primeros años de 
creación, lo que representa una limitada tasa de éxito en el entorno competitivo (Rodríguez, 2017), por lo que dentro de este contexto, las empresas del sector industrial tienen el deber de determinar los factores que obstaculizan en primera instancia su permanencia y posteriormente su crecimiento en el mercado competitivo, aun cuando cuentan con la peculiaridad de actuar conforme a las especificaciones de sus clientes (Suárez, Preckler, \& Farril, 2012).

Al respecto, se refieren datos de INEGI, que muestran que el Indicador Mensual de la Actividad Industrial (IMAI) del país disminuyó (-) $0.3 \%$ en términos reales en el último mes del año de 2019 respecto al del mes previo (INEGI, 2019), con base en cifras desestacionalizadas, como se muestra en la siguiente gráfica.

\section{Grafica 1. Actividad Industrial}

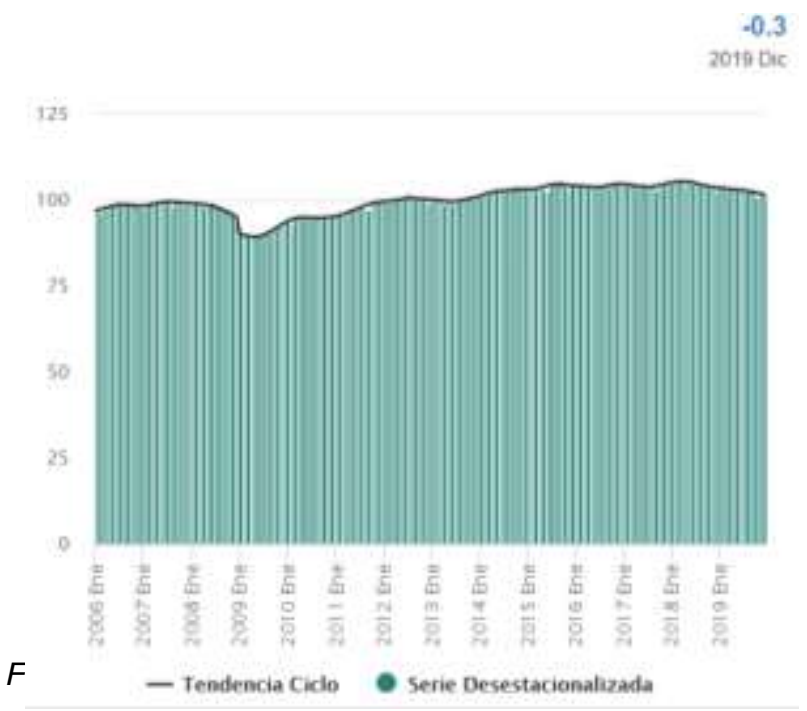

Como se observa, las PyMEs industriales enfrentan una serie de factores limitantes para competir en mercados internacionales, lo que las lleva a disminuir su grado de participación en el sector empresarial, y no obstante sus propiedades de crecimiento, los factores mencionados resultan un obstáculo inminente para su incorporación al entorno competitivo, por lo que deben considerarse como posibles restricciones a su competitividad.

\section{Principales restricciones que enfrentan las PyMEs}

Las PyMEs tienen una importancia significativa dentro de la economía de los países; las mejoras en su competitividad inciden directamente en su crecimiento y desarrollo, sin embargo, existen factores externos que imponen restricciones a este tipo de empresas y generan un entorno que no les es propicio, limitando sus perspectivas de crecimiento e impactan su competitividad.

\section{Tecnología e información}

De acuerdo a la dinámica del mercado, las PyMEs deben implementar cambios rápidamente, que les permitan la introducción y permanencia en el entorno competitivo; la tecnología es un medio para lograr este objetivo; sin embargo una de las principales restricciones para el crecimiento y desarrollo de las PyMEs mexicanas es la falta de acceso a tecnología de punta y/o tecnología que se adapte a sus capacidades productivas (Ycaza \& Sanchez, 2016), debido a que el costo de la tecnología es el factor limitante más importante para su adopción, además, debe tomarse en cuenta que la mayoría de estos negocios son familiares o surgen a través de la idea de un emprendedor, que en ocasiones no tiene los conocimientos idóneos para operar o adquirir equipos complejos.

Es importante destacar que las empresas que actualmente no incorporan la tecnología a sus procesos, no están del todo obsoletas, (Estrada, Cano, \& Aguirre, 2019) ya que existen empresas manufactureras artesanales, sin embargo, no tienen la misma capacidad para enfrentar las necesidades del mercado como las empresas que si la incorporan. Las ventajas de la incorporación de tecnología son muchas: a) disminución de costos de producción, b) menor incidencia de errores al mejorar la eficacia y la eficiencia de los trabajadores en la realización de los procesos y c) la optimización de la logística de distribución, entre otras, (Cuevas, 2018) sin embargo, la tecnología empleada por las PyMEs es las idónea.

Como ejemplo de lo anterior pueden señalarse los servicios de contabilidad. Es bien sabido que en grandes empresas existe un área específica destinada a estas tareas, se contratan los servicios de un especialista o bien se hace uso de un software contable, sin embargo, también se da el caso de empresas que no pueden pagar dichos servicios o comprar un software, incluso, no saben como usarlo. En esta situación se encuentran muchas PyMEs que, de acuerdo con estadísticas del INEGI, en un $38.4 \%$ hacen uso de un cuaderno para llevar la contabilidad de sus organizaciones, en tanto que el $23.6 \%$ de estas empresas ni siquiera realiza las tareas de contabilidad.

Por otro lado, el uso de Tecnologías de la Información y Comunicación (TIC) debe considerarse en este contexto, dado que las empresas de hoy, para ser competitivas deben de estar actualizadas. Esto se debe a que gran parte de los clientes tienen presencia en internet, al igual que sus competidores, sin embargo, el $7.6 \%$ de las medianas empresas y el $1.6 \%$ de las pequeñas, no tienen 
acceso a él (INEGI, 2019). Un ejemplo claro es la utilización de redes sociales como herramienta estratégica que aporta una fuente de datos acerca de las preferencias de los clientes y que, en la actualidad, ninguna organización debería desaprovechar, ya que es un medio para generar más ventas y hacer elaborar productos o servicios personalizados (Albornoz, Bastian , \& Failla, 2002).

Por último, cabe destacar que las PyMEs industriales en México son las que se ven más afectadas por esta restricción, debido a su constante necesidad de innovar en sus procesos o productos. (Calvo, 2018) Así, la tecnología no depende solamente de la compra de nueva y especializada tecnología como erróneamente se piensa, sino que la diferencia competitiva radica en qué tan eficientemente se gestionan, administran o dirigen los recursos que actualmente se tienen para generar conocimiento, investigación e innovación y así llegar al desarrollo de las capacidades dinámicas de la empresa (Mendoza, Chumbimuni, Rodríguez, \& Apaclla, 2001).

\section{Capital Humano}

El capital humano en las PyMEs es un factor determinante en el entorno competitivo. En función de los acelerados y constantes cambios en el desarrollo empresarial, se empiezan a generar nuevas necesidades de adquisición de personal calificado y experimentado que contribuya a la obtención de óptimos resultados para mantener la ventaja competitiva (Dessler y Varela citado en (Medina, Morales, \& Ulibarri, 2016). Gracias a las capacidades, ideas, habilidades y actitu des que el capital humano tiene, es como las empresas pueden captar y gestionar información para convertirla en una ventaja competitiva. Estos factores son fundamentales para alcanzar el éxito de las PyMEs, pues con un capital humano capacitado se pueden logras los objetivos y metas que éstas se plantean. (Castillo, 2012)

El capital humano puede ser dividido en dos categorías generales: a) gerentes y supervisores y b) administrativos y operativos, reconociendo para ambos casos la exigencia respecto a las competencias laborales (Zevallos, 2006), sin embargo, como se mencionó anteriormente, una de las principales características de las PyMEs es su reducido número de trabajadores, por lo que una de las primeras limitantes es la falta de una adecuada gestión del capital humano, lo cual puede dar lugar a que las actividades no se encuentren claramente definidas y que los trabajadores o directivos realicen tareas sin conocer exactamente cuál es el proceso o tomen malas decisiones que podrían incluso llevar a la empresa al fracaso (Barney citado en (Suárez \& Martín, 2006).
Por otro lado, en términos generales las PyMEs no consideran necesaria la implementación de consultoría o capacitación dentro de la organización, debido diversos factores entre los que destaca la elevada inversión que se requiere, limitando la capacitación a niveles técnicos, lo cual no siempre constituye la mejor opción. Adicionalmente, (López, Tricás, \& Toledano, 2012) se ha encontrado que el personal no solo carece de estas capacitaciones, sino que además tiene un nivel educativo muy bajo (Sánchez \& Castañeda citado en (Sapién, Piñón, \& Gutiérrez, 2014).

En contraposición, Ibarra (2010) señala que las empresas que han optado por capacitar a su personal, tienen mayores posibilidades de generar mejoras que faciliten su introducción a un mercado competitivo de alto nivel, y que cuando una empresa dentro de la clasificación de PyMEs cuenta con personal capacitado para hacer frente a sus retos, es cuando se comienza a desarrollar la innovación y en consecuencia, la integración al entomo competitivo.

\section{Innovación.}

La innovación consiste en buscar cambiar algo para hacerlo más productivo, desde crear un nuevo producto hasta generar algo para alcanzar procesos más eficientes (González, Rodríguez, \& González, 2019); no se limita a la adquisición de tecnologías, sino a aplicar la ciencia y la tecnología a la realidad, para resolver problemas en los mercados.

Esta actividad tiene muchos beneficios para la sociedad, sin embargo, lleva consigo un elevado grado de riesgo de fracasar y presenta también innumerables problemáticas.

Una investigación realizada por Chávez (2012) determina cuales son las principales problemáticas para la innovación en las empresas mexicanas, señalando las siguientes:

- El gobierno no prioriza la ciencia y la tecnología e incumple la meta de trabajar para llegar al $1 \%$ de inversión en investigación y desarrollo como proporción del PIB.

- Los empresarios se empeñan en obtener ganancias a corto plazo haciendo las cosas de la misma forma y basando su competitividad en la reducción de costos de los factores de la producción, en lugar de buscar una eficiencia conducida por la innovación y la inversión en tecnología y capital humano.

Lo anterior nos demuestra que en México existe una resistencia al cambio y a la innovación, debido a que muchos empresarios caen en la conformidad para desarrollarse además de carecer de una cultura de innovación (Cuevas, 2018). 
Las PyMEs hoy en día requieren invertir recursos para mejorar sus productos y/o servicios, debido al constante cambio de los mercados y de las necesidades de los consumidores. (Meixueiro, 2012) Se ha encontrado que las empresas que no se adaptan a estos cambios tienden a desaparecer antes de los primeros 10 años (CEPAL, 2018), atribuyendo su desaparición a la falta de planes estratégicos enfocados a la innovación, de cualquier forma, enfatizándose que cuando una empresa aspira al crecimiento, debe de adaptarse y cubrir las necesidades existentes.

Por otra parte, particularmente en la PyMEs industriales, la innovación representa un reto para su desarrollo, ya que de acuerdo al cambio tecnológico y el entono dinámico y complejo en el que se desarrollan, requieren estrategias competitivas en materia de innovación, elemento clave para su éxito competitivo.

\section{Financiamiento}

En lo que se refiere a la gestión del financiamiento para su desarrollo yt crecimiento, es sabido que gran parte de PyMES no consideran esta opción, lo que limita su crecimiento. Entre los principales factores que explican esta decisión de tomar financiamientos como alternativa para crecer se encuentran los siguientes:

- Altas tasas de interés,

- Falta de solvencia económica y escasas ganancias, que dificultan el hacer pagos derivados de financiamientos,

- Requisitos solicitados por la banca comercial para garantizar el préstamo (aval, otras garantías)

- Plazos de los créditos. Esto implica que los emprendedores, en función de la tendencia de nuevas empresas a desaparecer antes de cumplir los dos años de vida, en promedio, no tienen acceso a créditos a largo plazo.

Adicionalmente existen otros factores que influyen en el éxito o fracaso de las solicitudes de préstamos de PyMEs, como son: el propósito del préstamo y la estructura legal de la empresa, es decir, la mayor parte empresas solicitan préstamos para activo circulante, considerado como pago a corto plazo, con escasas posibilidades de que los bancos lo otorguen. Sin embargo, si el concepto por el cual se está solicitando el préstamo es para invertir en activos se tiene mayor posibilidad de obtenerlo. (Cowling y Mitchell citado en (Gomez \& Garcia, 2009).
En este mismo orden de ideas, algunas encuestas revelaron que las PyMEs en México enfrentan restricciones al crédito no importan do su costo, aunque un porcentaje de $31.7 \%$ de las empresas encuestadas reportaron que no solicitan créditos bancarios debido a las altas tasas de interés. (Gomez \& Garcia, 2009). Es por esta razón que la principal fuente de financiamiento de estas empresas sean los proveedores, quienes cubren gran parte de su financiamiento. Como ejemplo se señala que tan solo en 2007 se presentaron registros de financiamiento a PyMEs por parte de proveedores en un $71 \%$, decayendo hasta $62.6 \%$ en el año de 2008 . Si bien la causa no es muy precisa, se estima que dicha caída se debe a pérdida de liquidez de las empresas proveedoras de materia prima (Lecuona, 2009).

Otro aspecto a considerar es que muchas empresas no tienen conocimiento de los apoyos que el gobierno otorga. Uno de los programas más relevantes, que estuvo vigente todavía hasta el año de 2019 era el del Institu to Nacional del Emprendedor (INADEM), encargado de apoyar a las PyMEs para el desarrollo de sus proyectos. Este programa contaba con diversas categorías que apoyaban a la inserción en el mercado, mediante la puesta a disposición de incubadoras de empresas, programas de sensibilización para fomentar el emprendimiento, asesoría para acceder a un financiamiento, y fortalecimiento de intermediarios financieros no bancarios, con el propósito que las empresas micro, pequeñas y medianas contaran con diferentes opciones de financiamiento (INADEM, 2012).

No obstante, estos apoyos de financiamiento, que contribuían a minimizar las restricciones al financiamiento, en muchas ocasiones no se contaba con la respuesta de emprendedores o PyMEs, aun cuando de acuerdo al tipo de proyecto propuesto, debiera pagarse sólo un porcentaje de lo obtenido en financiamiento. Infortunadamente con el cambio de gobierno federal a finales del año de 2019, se anunció la desaparición del INADEM, anunciando que sería sustituido por otro programa de apoyo a PyMEs.

Uno de los agentes de apoyo al financiamiento de las PyMEs es el Consejo Nacional de Ciencia y Tecnología (CONACYT), centrado en el financiamiento de proyectos de base tecnológica. Este programa apoya en distintas etapas, además de proveerles de Inversión temporal de capitales para impulsar empresas basadas en conocimientos científicos y tecnológicos en su etapa de despegue. (Saavedra \& Tapia, 2011)

De acuerdo a lo expresado por el gobierno federal, durante el año de 2020 se desarrollarán programas en sustitución, sin embargo no existe claridad, dado que 
dentro del Presupuesto de Egresos para este año se aborda de manera limitada el apoyo de financiamiento a las PyMEs: a) financiamiento para PyMEs del sur-este, con tasa preferencial, b) créditos a MIPyME; mediante programa de garantías con la banca privada se buscará apoyar a más de 130 mil pequeñas y medianas empresas y por último, se menciona que se apoyará la creación y consolidación de las MIPyMEs productivas y las personas emprendedoras al lograr que accedan a las cadenas globales de valor a través de la proveeduría nacional, el acceso al capital físico y financiero, la capacitación del capital humano y a la innovación de sus procesos (SHCP, 2019)

En definitiva, las restricciones para el financiamiento en México es un tema no resuelto, considerando que con el periodo de gobierno precedente existían mayores probabilidades de obtener un crédito a tasas más bajas, en tanto que con el gobierno actual se observa que algunos de los apoyos se canalizan a la región del su reste, el financiamiento se otorga a PyMEs con más de 4 años operando, y no se otorgan apoyos para la apertura de nuevas PyMEs

\section{Política Publica}

En cuanto a las políticas públicas para PyMEs dentro del país, se orientan a apoyos a la vinculación, innovación de las organizaciones, financiamiento a proyectos $y$ capacitaciones. Aunque se han realizado trabajos sobre políticas públicas que apoyen a las PyMEs, todavía existen restricciones que afectan al desarrollo de estas empresas. Entre dichas restricciones se encuentra la exportación de productos por parte de PyMEs mexicanas constituye una restricción no mitigada, en tanto que a la fecha de celebración del TLC, el gobierno de México no contaba con un programa de apoyo al desarrollo de estas empresas, sino hasta después de 10 años, cuando en el año de 2002 fue publicada en el Diario Oficial de la Federación la Ley para el Desarrollo de la Competitividad de la Micro, Pequeña y Mediana Empresa, con el objetivo de "promover el desarrollo económico nacional a través del fomento a la creación, de micro, pequeñas y medianas empresas y el apoyo para su viabilidad, productividad, competitividad y sustentabilidad (Gomez L. , 2016)".

Sin embargo, los programas que se han desarrollado en el país no son suficientes, ya que los tratados celebrados por México para fomentar la competitividad han desatado contradicciones y controversias que afectan a la economía de las PyMEs, debido principalmente a que con los tratados, así como se pueden exportar productos mexicanos, se importan productos extranjeros, la diferencia estriba en que muchas de las veces los productos mexicanos son rechazados por no contar con los certificados que los países requieren, en tanto que en México aceptan productos incluso sin certificados, lo que redunda en perjuicios para las PyMEs mexicanas pues sus productos únicamente tienen posibilidades de ser vendidos en el mercado nacional, en donde además compiten con empresas extranjeras con mayor reconocimiento. Adicionalmente, los aranceles que se aplican en los productos mexicanos son altos, lo que trae como consecuencia que al poner los productos en venta en otros mercados estos eleven sus precios. (Palacios, 2013)

No obstante, en el Plan Nacional de Desarrollo (PND) 2013-2018 se mostraban estrategias y líneas de acción para democratizar la productividad, con el principal objetivo de eliminación de restricciones, para todos los sectores. (Plan Nacional de Desarrollo, 2013-2018)

Por otra parte, se encuentra también la falta de apoyo por parte de gobierno para regularizar la entrada de empresas extranjeras al país, que por prestigio de marca gana mercado dejando a las PyMEs sin margen para posicionarse dentro del mismo.

Sin duda esto ha generado mayores impactos y consecuencias negativas para las empresas de giro industrial, ya que si no abarcan el mercado deseado, tienden a desaparecer, con la consecuente reducción o pérdida de empleos, razón por la que es de vital importancia regular esta situación (Saavedra, Tapia, \& Aguilar, 2013).

Como puede observarse, hasta la fecha se encuentran restricciones para las PyMEs, y aunque se han planeado políticas para erradicarlas, los logros no son significativos puesto que el mercado nacional prefiere comprar artículos importados y de menorcalidad, en perjuicio a la economía de las PyMEs.

\section{Conclusiones}

A manera de conclusión puede establecerse que la supervivencia de las PyMEs industriales está ligada directamente a la competitividad y a la facilidad de adaptación a los mercados dinámicos, donde conceptos como la personalización de los productos y servicios, consumidor responsable e interconectividad, entre otros, toman un papel preponderante y abren nuevos nichos de mercado en los cuales estas empresas pueden asegurar su supervivencia y consolidación.

Sin embargo, entrara este tipo de mercados y atender sus propuestas representa un reto importante para la industria en general sin importar el mercado, su tamaño o 
nacionalidad, por lo que se precisa que las empresas interesadas en cubrir estas demandas realicen un diagnóstico interno que les permita identificar sus restricciones tecnológicas, de financiamiento y capital humano, con el propósito de maximizar las oportunidades y fortalezas derivados, y reducir sus debilidades, con mayores probabilidades de enfrentar las amenazas existentes.

Debe considerarse también que las políticas públicas definidas como mecanismos para orientar el curso de agentes específicos, requieren ser reformuladas para atenderde manera precisa las necesidades de las PyMES industriales y con ello lograr que se encuentren preparadas para competir en mejores condiciones en un mercado altamente globalizado, ya que en gran medida, al lograr un éxito sostenido se logrará también consolidar la economía Mexicana y generar un crecimiento y desarrollo económico sostenido.

\section{Referencias}

Albornoz, P., Bastian, E., \& Failla, F. (2002). Tecnologias de informacion en la pqueña y mediana empresa y el papeldel estado. Seminario para Optar de ingeniero en informacion y control de gestion. Chile : Facultad de ciencias economicas y administra tivas.

Arroyo, B., Espinosa, R., \& Erazo, F. (2011). Diagnóstico de las PYME del Sector Industrial en el Estado de Guanajuato, México y su Visión de Negocios.

Cabrera, A., P, L., \& Ramirez, C. (2011). La competitividad empresarial: Un marco conceptual para su estudio. Documentos de investigación, 4 , $8-54$ Obtenido https://papers.ssrn.com/sol3/papers.cfm?abstract_id=2016597

Calvo, O. (2018). La Gestión del Conocimiento en las Organizaciones y las Regiones: una Revisión de la Literatura. Tendencias. Tendencias, I(19), 140-163. doi:http://dx.doi.org/10.22267/rtend.181901.91

Canales, V., \& Arnaldo, L. (2013). Manualde planificación estratégica para la pequeña y mediana empresa (PYME) pertenecias a la Cámara de Comercio de Chillán. Facultad de ciencias empresariales, 176 Obtenido http://repobib.ubiobio.cl/jspui/bitstream/123456789/958/1/Venegas\%2 0Canales\%2c\%20Luis\%20Arnaldo.pdf

Cardozo, E., Velasquez, d. N., \& Rodriguez, M. (2012). 6th International Conference on Industrial Engineering and Industrial Mana gement. La definición de PYME en América: Una revisión del estado del arte Definition of SMEs in Latin America, (págs. 1345-1352). Obtenido de 6th International Conference on Industrial Engineering and Industrial Management.

Castillo, R. (2012). Desarrollo del capital humano en las organizaciones. México: Red tercer milenio. Obtenido de http://www.aliat.org.mx/BibliotecasDigita les/economico_administrativ o/Desarrollo_del_capital_humano_en_las_org.pdf

CEPAL . (2018). Mipymes en America Latina: un frágil desempeño y nuevos desafíos para las políticas de fomento. Mejores políticas para las micro.
Chávez, M. (2012). Innovación en México: principales problemas. México: CESOP. Obtenido de http://congreso.investiga.fca.unam.mx/docs/xvi/docs/1W.pdf

Cuevas, H. (2018). El impacto de la tecnología de equipo en las pymes manufactureras mexicanas. Revista Facultad de Ciencias Contables, Económicas y Administrativas-FACCEA, 1(8), 43-54.

Diario Oficial de la Federación. (2016). Secretaria de Economia. Obtenido de Instituto Nacionaldel Emprendedor.

Diaz, L. (2006). La competitividad...i a qué se refiere?. Ensayos de economia, 16(29), 107-117.

Dussel, E. (2004). Pequeña y mediana empresa en México: condiciones, relevancia en la economía y retos de política. Scielo, l(2). Obtenido de $\mathrm{http}: / /$ www.scielo.org.mx/scielo.php?script=sci_arttext\&pid=S1665952X2004000200006

Estrada, S., Cano , K., \& Aguirre, J. (2019). ¿Cómo se gestiona la tecnología en las pymes? Diferencias y similitudes entre micro, pequeñas y medianas empresas. Contaduria y administracion, 64(SPE1). doi:10.22201/fca.24488410e.2019.1812

Gomez, A., \& Garcia, S. (2009). Restricciones a la financiación de la PYME en México: una aproxima ción empírica. analisiseconomico, 217-238.

Gomez, L. (2016). a Gestión de la Competitividad en la MIPYME Mexicana: Diagnóstico Empírico desde la Gestión del Conocimiento. Revista de administracion de UNIMEP, 183-186.

Gonzalez, C., \& Herrera, J. (2011). Pymes mexicanas, incubadoras del desarrollo y crecimiento económico del México actual. Hablemos de Economia, 45.

González, J., Rodríguez, M., \& González, O. (2019). Factores que inciden en la gestión de conocimiento y la innovación abierta en empresas colombianas. Revista Virtual Universidad Católica del Norte(58), 116138. doi:10.35575/rvuen.n58a10

Heradia, E. (2014). Las PYMES en México: desarrollo y competitividad. Observatorio de la Economía Latinoamericana, 201. Obtenido de https://scholar.google.com/scholar_url?url=http://www.eumed.net/curs econ/ecolat/mx/2014/cooperacion.zip\&hl=es\&sa=T\&oi=gsbgop\&ct=res\&cd $=2 \& d=596845676790886099 \&$ ei $=$ YoRqXrT7EM ZywSmy53wDA\&scisig=AAGBfm0PBJEn33HtpTEjaFnFr8MKycR WOQ

Ibarra , M., \& Gonzalez, L. (2010). La flexibilidad laboralcomo estrategia de competitividad y sus efectos sobre la economía, la empresa y el mercado de trabajo. Contaduría y administración (231),33-52. Obtenido de http://www.scielo.org.mx/pdf/cya/n231/n231a3.pdf

INADEM. (2012). Matriz de vinculación de categorías y convocatorias con los ejes rectores de la planeación nacional 2013-2018.

INEGI. (2018). Encuesta Nacional sobre Productividad y Competitividad de las Micro, Pequeñas y Medianas Empresas (ENAPROCE) 2018. Obtenido https://www.inegi.org.mx/programas/ena proce/2018/default.html\#Tab ulados

INEGI. (Diciembre de 2019). El INEGI informa que el Indicador Mensual de la Actividad Industrial (IMAI) del país disminuyó (-)0.3\% en términos reales en el último mes de 2019 respecto al del mes previo, con base en cifras desestacionalizadas. Obtenido de Datos INEGI: https://www.inegi.org.mx/temas/imai/

INEGI. (2019). INEGI presenta resultados de la encuesta nacional sobre productividad y competitividad de las Micro, Pequeñas y Medianas empresas (. México: INEGI. Obtenido de https://www.inegi.org.mx/contenidos/saladeprensa/boletines/2019/espe cia les/ENAPROCE2018.pdf

International Finance Corporation. (2018). Pymes mexicanas, un panorama para 2018. Obtenido de International Finance Corporation: https://www.forbes.com.mx/pymes-mexicanas-un-panorama-para2018/ 
Lecuona, R. (2009). El financiamiento a las Pymes en México: la experiencia reciente. Economia UNAM, 69-91.

López, L., Tricás, J., \& Toledano, R. (2012). Principales prácticas de recursos humanos de las PyMEs industriales exitosas. Universidad \& Empresa, 23, 19-43. Obtenido de https://www.redalyc.org/articulo.oa? $\mathrm{id}=187226257007$

Martinez, A., \& Mejia, M. (2013). Financiación y factores competitivos de la Pyme industrial de México: un estudio empírico. (Doctoral dissertation, Benemérita Universidad Autónoma de Puebla-México).

Medina, M., Morales, M., \& Ulibarri, H. (15 al 18 de Noviembre de 2016). Impacto del capital humano como factor de competitividad en la empresa manufacturera en Yucatan. Obtenido de UNAM: http://ru.iiec.unam.mx/3427/1/178-Medina-Morales-Ulibarri.pdf

Meixueiro, G. (2012). Retos para impulsar la innovación en México. México: CESOP. Obtenido de http://congreso.investiga .fca.unam.mx/docs/xvi/docs/1W.pdf

Mendoza, F., Chumbimuni, F., Rodríguez, R., \& Apaclla, V. (2001) Gestión de la innovación y tecnología en las empresas peruanas del sector retail.

OCDE. (1992). Technology and the Economy: The Key Relationships. Paris: Autor.

Palacios, P. (Mayo de 2013). Desempeño Exportador e Innovador de la Pyme Mexicana como estrategias de internacionalización. Tesis. Madrid, España: Universidad Autonoma de Madrid

Pazos, D., \& Lopez, M. (2007). La innovación como factor clave en la competitividad empresarial: un estudio empírico en pymes. Revista Galega de Economía, 16(2).

Plan Nacional de Desarrollo. (2013-2018). Plan Nacional de Desarrollo 2013-2018. Obtenido de Sistema Nacionalde Información Estadística y Geográfia:

https://www.snieg.mx/contenidos/espanol/normatividad/MarcoJuridico /PND_2013-2018.pdf

Porter, M. (1991). La ventaja competitiva de las naciones (Vol. 1025). Buenos Aires.

Rivas, L. (2002). Nuevas formas de organizacion. Scielo , 18(82), 13-45. Obtenido http://www.scielo.org.co/scielo.php?script=sci_arttext\&pid=S0123 5923200200010000

Rodriguez, A. (2015). La competitividad y los factores del exito de las PyMES. Revision teórica. Guarracuco, 113(12). Obtenido de http://revistas.unimeta.edu.co/index.php/rc_es_guarracuco/article/view $/ 154$

Romero, I. (2006). Las PYME en la economía global. Hacia una estrategia de fomento empresarial. Problemas del desarrollo, 37(146), 31-50.

Ruiz, C. (2013). Estrategias empresaria les más utilizadas por las pymes en Sincelejo. Economicas CUC, 34(1), 183-202. Obtenido de http://revistascientificas.cuc.edu.co/index.php/economicascuc/article/vi ew/583

Saavedra, G., \& Milla, S. \&. (2012). Determinación de la competitividad de la PYME en el nivel micro: El caso de del Distrito Federal, Mexico. Faedpyme. International Review (Steubenville, Ohio), 2(4), 23-33.

Saavedra, M., \& Tapia, B. (2011). Las mejores prácticas en la Mipyme Industrial Mexicana. México: UNAM. Obtenido de http://congreso.investiga .fca.unam.mx/docs/xvi/docs/1W.pdf

Saavedra, M., Tapia, B., \& Aguilar, M. D. (2013). El impacto de las políticas públicas en la mipyme mexicana. Ciencias Administrativas, 1 19

Sapién, A., Piñón, L., \& Gutiérrez, M. (2014). Capacitación en la empresa mexicana: un estudio de formación en el trabajo. Civilizar, 123-134 Obtenido de http://www.scielo.org.co/pdf/ccso/v14n27/v14n27a09.pdf
SHCP. (2019). criterios generales de politica publica para la iniciativa de ley de ingresos y proyecto de presupuesto de egresos de la federacion correspondientes al ejercico fiscal 2020. México.

Suárez,L., Preckler, J., \& Farril, R. (2012). Principales prácticas de recursos humanos de las PyMEs industriales exitosas. Universidad \& Empresa, 14(23), 19-43.

Suarez, L., Tricas, J., \& Toledano, R. (2012). Principales prácticas de recursos humanos de las PyMEs industriales exitosas. Universidad y empresa, 14(23), 19-43. Obtenido de https://www.redalyc.org/pdf/1872/187226257007.pdf

Suárez, T., \& Martín, M. (2006). Impacto de los capita les humano y organizacionalen las estrategias de la PYME. Scielo, 229-248. Obtenido de http://www.scielo.org.co/pdf/cadm/v21n35/v21n35a10.pdf

Ycaza, D., \& Sanchez, A. (2016). Obstáculos para la micro, pequeña y mediana empresa en América Latina. Pymes, Innovación y Desarrollo,, $4(2), 21-36$.

Zevallos , E. (2006). Obstaculos al desarrollo de las pqueñas y medianas empresas en amercia latina. Journal of Economics, Finance and Administrative Science, $\quad 11(20), \quad 75-96 . \quad$ Obtenido de https://www.redalyc.org/pdf/3607/360735259004.pdf 